Revista Destaques Acadêmicos, Lajeado, v. 9, n. 4, 2017. ISSN 2176-3070 DOI: http://dx.doi.org/10.22410/issn.2176-3070.v9i4a2017.1670 www.univates.br/revistas

\title{
COMPARAÇÃO DE DESEMPENHO DAS ARQUITETURAS SOC DE CPU ARM CORTEX A57 E INTEL X86 COMO SERVIDORES DOS SOFTWARES APACHE E NGINX
}

\author{
Mateus da Silva ${ }^{1}$, Ronaldo Husemann ${ }^{2}$, Marcelo de Gomensoro Malheiros ${ }^{3}$
}

Resumo: A evolução dos processadores tornou possível a utilização de CPUs modernas em servidores web, os que exigem além do atendimento a grande número de requisições simultâneas, cada vez mais exigem soluções de hardware eficientes em termos de consumo de energia. Na prática, chips energeticamente eficientes devem garantir alto desempenho com baixas demandas de dissipação térmica e consumo de potência. Visando este tipo de aplicação, foi lançada em 2015 a arquitetura ARM Cortex A57 que une reduzido consumo com recursos específicos para servidores. Alternativamente a empresa Intel também introduziu linhas de processadores de baixa potência como Avoton e Braswell para o mesmo propósito. Considerando estas duas proeminentes tecnologias, este trabalho realizou diversos testes praticos para mensurar o desempenho e o consumo de energia das duas plataformas ARM e Intel x86, mais particularmente representados pelos processadores Nvidia Tegra X1 e Pentium Braswell N3700 respectivamente. $\mathrm{O}$ objetivo principal é comparar as CPUs em termos de desempenho e consumo de energia quando operando como servidores HTTP Apache e NGINX. Os benchmarks obtidos indicaram que o processador ARM Tegra X1 tem marginalmente melhor desempenho, enquanto o CPU Intel x86 Pentium N3700, em termos de consumo de energia, a CPU é mais eficiente.

Palavras-chave: CPU. Eficiência energética. ARM. Intel x86.

1 Bacharel em Engenharia da Computação pela Universidade do Vale do Taquari, UNIVATES. E-mail: mateusdsrs@gmail.com

2 Mestre e Doutor em Engenharia Elétrica pela Universidade Federal do Rio Grande do Sul, UFRGS. Professor adjunto do Departamento de Engenharia Elétrica da UFRGS. E-mail: rhusemann@inf.ufrgs.br

3 Mestre em Engenharia Elétrica pela Universidade Estadual de Campinas e doutor em Computação pela Universidade Federal do Rio Grande do Sul, UFRGS. Professor do centro de ciências exatas da UNIVATES. E-mail: mgm@univates.br 


\section{INTRODUÇÃO}

Com a evolução dos serviços de internet (web 2.0, centro de dados em nuvem e big data) cada vez mais se exige o aperfeiçoamento do desempenho e consumo energético dos processadores empregados nos servidores. Focando em aumento da eficiência energética a Intel lançou em 2013 uma família de processadores chamada Avoton que opera com TDP (Thermal Design Power) máximo de $20 \mathrm{~W}$ com até 8 núcleos de processamento operando a $2.4 \mathrm{GHz}$ (INTEL, 2013).

Em 2015 a Intel passou a produzir processadores com consumo energético ainda menor, TDP de cerca de $6 \mathrm{~W}$, em uma linha chamada Braswell (INTEL, 2015). A título de comparação, pode-se considerar o processador Intel E7-4820v2, com 8 núcleos, que tem um TDP de 105W (consumo de energia várias vezes superior) (INTEL, 2016). Paralelamente aos avanços da Intel, a empresa ARM apresentou em 2015 a microarquitetura Cortex A57 como alternativa para o mercado de servidores enfatizando desempenho e eficiência energética (ARM, 2016). A arquitetura ARM Cortex A57 possui suporte a 64 bits permitindo o uso de mais de 4 GB de memória RAM e instruções de virtualização por hardware.

Ambos os chips Intel e ARM citados possuem a característica de serem SOC (System on Chip) possibilitando o desenvolvimento de servidores com hardware de tamanho reduzido (ANTHONY, 2012).

Para avaliar o desempenho da microarquitetura Cortex A57 perante a tecnologia Intel Braswell x86 este artigo apresentará ensaios de desempenho e consumo energético dos processadores buscando avaliar de forma prática as duas arquiteturas, como servidores HTTP Apache e NGINX que representam os softwares mais utilizados nesse segmento (NETCRAFT, 2016).

Alguns pesquisadores analisam o desempenho de arquiteturas SOC para o mercado de servidores, enfatizando a comparação de arquiteturas ARM em relação à Intel x86. Como exemplo pode-se citar os trabalhos de Aroca e Gonçalves publicado em 2012 e Blem e Menon de 2013.

São trabalhos relevantes, porém considerando o cenário atual do mercado de microprocessadores SOC, percebe-se que os resultados destes trabalhos se encontram defasados. Esta situação motivou o presente trabalho no sentido de avaliar tecnologias SOC modernas (estado da arte) para verificar a real adequação destas ao cenário de servidores web.

Os processadores utilizados em testes de comparação foram Pentium Braswell N3700 e Nvidia Tegra X1. Os resultados obtidos apontaram que o SOC ARM Nvidia Tegra X1 possui desempenho médio $12 \%$ superior ao chip Intel Pentium N3700 em todos os testes, enquanto o processador Intel se demonstrou $19 \%$ mais eficiente energeticamente. 


\section{ESTADO DA ARTE}

Essa seção descreve detalhes das tecnologias de processadores SOC e softwares empregados correntemente como servidores nos ensaios e discussão dos mesmos.

Chama-se de SOC um chip que integra em uma pastilha todas suas funcionalidades, tais como CPU, controladoras de disco, rede, vídeo e outras funções. Entre as vantagens da tecnologia SOC estão a redução da placa lógica do sistema decorrente do menor número de CIs e o menor consumo energético pelo alto nível de integração (ANTONY, 2012).

A seguir se apresentam duas tecnologias emergentes de processadores SOC para o mercado de servidores.

\subsection{Nvidia Tegra X1}

O microprocessador Nvidia Tegra $X 1$, fabricado na tecnologia de 20 nanômetros, implementa a tecnologia ARM Big Litle que incorpora 8 núcleos, sendo 4 da microarquitetura Cortex A57 para alto desempenho e 4 núcleos Cortex A53 especializados em baixo consumo energético (NVIDIA, 2015). A tecnologia Big Litle alterna entre os núcleos A57 e A53 conforme a demanda do sistema, ativando somente 4 núcleos simultâneos; o chip Tegra X1 possui TDP de $10 \mathrm{~W}$ (SMITH, 2015). O chip também incorpora controlador de memória LPDDR4, GPU Nidia Maxwell de 256 núcleos, CODEC de vídeo em hardware e outros periféricos como visto a seguir (NVIDIA, 2015).

Figura 1 - Arquitetura interna Nvidia Tegra X1

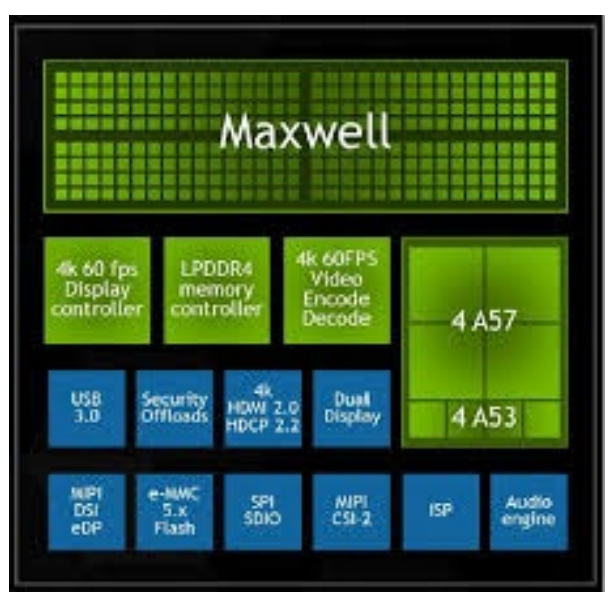

Fonte Nvidia (2015). 


\subsection{Intel Pentium N3700}

O Pentium N3700 (FIGURA 2) é um SOC baseado na microarquitetura Intel Braswell, contendo 4 núcleos de CPU que operam conforme demanda na faixa de frequência de $1.6 \mathrm{GHz}$ até $2.4 \mathrm{GHz}$ o que garante TDP médio de $6 \mathrm{~W}$. O chip N3700 é compatível com memória DDR3 de $1600 \mathrm{MHz}$, além de suportar as instruções x86-64 e instruções de virtualização Intel VT-x. Os quatro núcleos de CPU compartilham cache L2 de 2 MB (INTEL, 2015).

Figura 2 - Chip Braswell

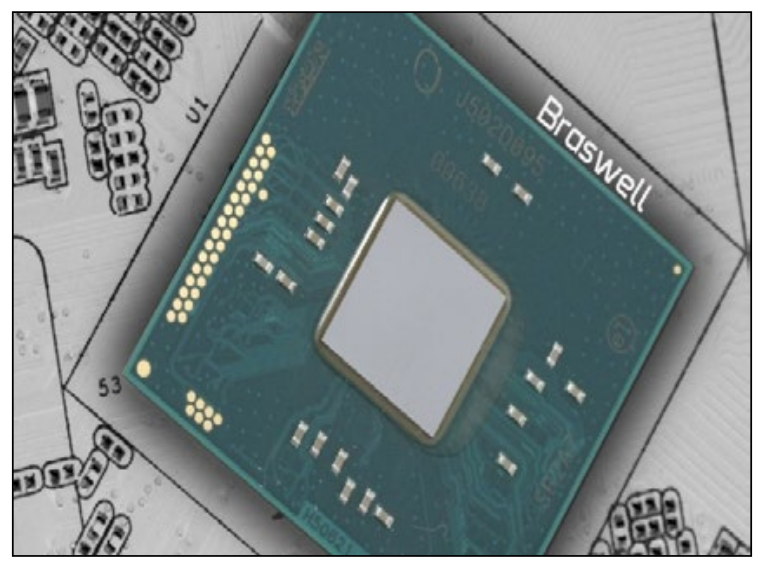

Fonte Intel (2015).

\subsection{Servidores Apache e NGINX PHP}

As principais funcionalidades dos servidores HTTP são receber, processar e enviar ao cliente respostas de requisições de páginas de internet. Mensurar o desempenho de um servidor HTTP é relevante para verificar a eficiência do sistema computacional que o executa (AROCA; GONÇALVES, 2012).

Porém são vários os aplicativos de servidores disponíveis no mercado hoje para atender a estas tarefas. Considerando isto, no presente trabalho foram escolhidos dois softwares de servidores diferentes: Apache e NGINX.

A escolha de servidores distintos permitiu verificar se havia diferença no desempenho dos dois processadores ao executar essa tarefa, cuja disparidade poderia ser proveniente de codificação do programa e compilação com menor otimização para uma plataforma ou outra, além da implementação do suporte a multithread de cada um.

Com objetivo de utilizar mais processamento ao atender as requisições HTTP o Apache e o NGINX foram configurados com o modulo PHP com a 
finalidade de realizar os testes com páginas geradas dinamicamente para aumentar o uso de CPU por cada requisição HTTP atendida.

\subsection{Trabalhos relacionados}

Os autores Aroca e Gonçalves (2012) fizeram ensaios de desempenho em vários processadores de arquitetura ARM e x86 com a finalidade de analisar desempenho e eficiência energética dos mesmos. Os processadores x86 estudados foram Turion MK-38, Atom N280 e Xeon X3450 e os CPUs de arquitetura ARM OMAP 4430 e OMAP 3730. Os testes demonstraram que o processador ARM OMAP 4430 foi mais eficiente atendendo aproximadamente 300 requisições por (W.s), o Atom N280 apresentou o melhor resultado da arquitetura x86 resolvendo aproximadamente 150 requisições por (W.s). Em relação ao desempenho o processador ARM OMAP 4430 processou 1448 requisições por segundo enquanto o Atom N280 resolveu 926, resultando em desempenho $56 \%$ superior para o OMAP 4430.

Blem e Menon (2013) realizaram uma comparação prática entre CPUs ARMv7 (Cortex A8 e A9) e Intel x86 (i7 Sandbridge 2700 e Atom N450). Experimentos avaliaram parâmetros como desempenho, eficiência energética e número de instruções. Para melhor comparar os resultados foram normalizados. Concluiu-se que a CPU A8 obteve melhores resultados em termos de potência, seguida pelas A9 e N450 e por último o i7 2700. Em termos de desempenho/ potência energética as A9 e N450 se mostraram bem similares sendo que i7 atingiu os melhores resultados.

\section{EXPERIMENTOS PRÁTICOS}

Esta sessão visa detalhar os experimentos utilizados para parametrizar os ensaios com os dois processadores testados. Na parte de hardware há informações dos sistemas computacionais x86 e ARM utilizados. Também está descrito a metodologia e materiais utilizados para mensurar o consumo de energia dos computadores.

\subsection{Características dos processadores avaliados}

Os processadores Nvidia Tegra X1e Pentium N3700 possuem algumas semelhanças técnicas. A velocidade de operação dos dois é próxima, apesar da velocidade de operação do Pentium não ser fixa variando de 1.6 a $2.4 \mathrm{GHz}$, mas pode se considerar $2 \mathrm{GHz}$ como intermediário sendo próximo do clock de 2.13 GHz do Tegra X1. O TDP do Pentium é de $6 \mathrm{~W}$ contra os $10 \mathrm{~W}$ do X1, a diferença de $40 \%$ é considerável, porém não deve influenciar tanto nas avaliações feitas que deve focar mais na eficiência do que no consumo energético bruto.

Ambos os SOCs possuem 4 núcleos de CPU (levando em conta que somente os núcleos Cortex A57 de alto desempenho foram utilizados) 
compartilhando $2 \mathrm{MB}$ de cache se segundo nível. Os dois processadores possuem três unidades de execução fora de ordem, suporte a instruções SIMD e contêm registradores de 64 bits (INTEL, 2015; ARM, 2015).

\subsection{Plataforma ARM}

Como ambiente para executar os testes de desempenho e consumo de energia no SOC Tegra X1 foi utilizada a plataforma Nvidia Shield TV (FIGURA 3). Esta plataforma é equipada com 3 GB de memória RAM LPDDR4 operando a $3200 \mathrm{MHz}, 16 \mathrm{~GB}$ de armazenamento flash interno, porta gigabit ethernet, 2 USB padrão 3.0 e slot para cartão SDHC (NVIDIA, 2016). Para os experimentos foi acionado ao Shield TV um cartão SDHC Kingston de 32 GB (KINGSTON, 2016) que suporta $90 \mathrm{MB}$ de leitura e $45 \mathrm{MB}$ de gravação; o qual serviu para armazenamento do sistema operacional e demais softwares.

Figura 3 - Nvidia Shield TV

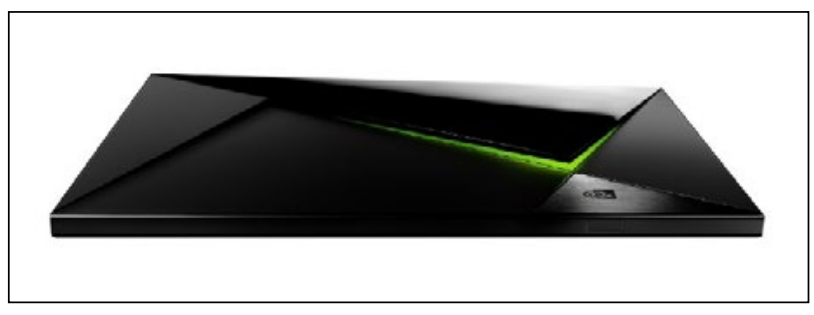

Fonte: Annadtech (2015).

\subsection{Plataforma Intel x86}

A plataforma x86 escolhida foi composta pela placa mãe mini ITX Asrock N3700 que possui o SOC Intel Pentium N3700, 4 portas SATA 3, 6 USB 2.0, 2 USB 2.0, porta ethernet gigabit e suporte até 16 GB de memória RAM DDR 3 (ASROCK, 2015). O computador foi configurado com 2 módulos de memória RAM DDR $31600 \mathrm{MHz}$ de 2 GB totalizando 4 GB. O sistema operacional e os programas foram instalados em uma unidade flash USB 3.0 de 64 GB com velocidade de leitura de $150 \mathrm{MB} / \mathrm{s}$ e $60 \mathrm{MB} / \mathrm{s}$ de escrita (LEXAR, 2016). 


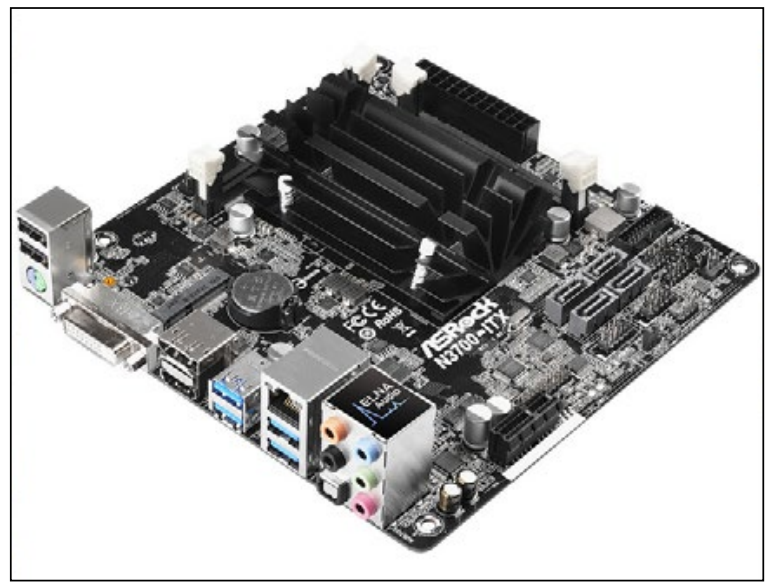

Fonte: Asrock (2016).

Tabela 1 - Comparativo da configuração das plataformas testadas

\begin{tabular}{l|c|c}
\hline Característica & Asrock N3700 & Nvidia Shield TV \\
\hline CPU & Pentiun N3700 4 núcleos & Tegra X1 4 núcleos \\
& 1.6 a $2.4 \mathrm{GHz} 2 \mathrm{MB}$ de L2 & $2,13 \mathrm{GHz} 2 \mathrm{MB}$ de L2 \\
\hline Memória & $4 \mathrm{~GB}$ DDR 3 $1600 \mathrm{MHz}$ & $\begin{array}{c}3 \mathrm{~GB} \mathrm{LPDDR} 42400 \\
\mathrm{MHz}\end{array}$ \\
\hline Armazenamento & $\begin{array}{c}\text { Flash USB 3.0 } 150 \mathrm{MB} / \mathrm{s} \text { leitura } \\
90 \mathrm{MB} / \mathrm{s} \text { escrita }\end{array}$ & $\begin{array}{c}\text { Flash cartão SD 90 } \\
\mathrm{MB} / \mathrm{s} \text { leitura 45 MB/s } \\
\text { escrita }\end{array}$ \\
\hline
\end{tabular}

Fonte: Asrock (2015) e Nvidia (2016).

\subsection{Configuração de software}

O sistema operacional adotado nas duas plataformas foi o Ubuntu 16.06 Xenial Xerus, pelo fato que possui versão compatível de 64 bits com os SOCs utilizados. O Ubuntu foi configurado nos dois computadores somente com serviços essenciais para otimizar os recursos do hardware disponível. Com a finalidade de reduzir a latência de leitura dos dados acessados pelos programas Apache e NGINX foi utilizado o recurso RAMFS do kernel do Linux para criar uma partição virtual em memória de 400 MB, através deste método foi eliminado as influencias dos acessos USB e cartão SD nos experimentos. 
O suporte à linguagem PHP na versão 7.0 foi configurado nos programas Apache versão 2.4.18 e NGINX versão 1.10.

\subsection{Ferramentas de benchmark}

Com o propósito de mensurar o desempenho dos servidores escolhidos, optou-se por utilizar o PHP Benchmark Script (TOYOTARO, 2008) como a página acessada durante os testes.

Durante cada acesso HTTP ao Script o programa executa milhares de operações matemáticas, manipulação de strings e laços de repetições. Esse método foi escolhido, pois assim a utilização do PHP Benchmark Script (TORRIS, 2012) faz uso intenso de CPU durante o processamento das operações executadas pelo programa.

Em média a plataforma ARM levou aproximadamente 2,5 segundos para executar um acesso ao PHP Benchmark Script enquanto que o processador N3700 levou aproximadamente 2,9 segundos. Para verificar o desempenho e consumo energético do sistema x86 e ARM foram efetuados ensaios com 4 cargas diferentes como pode ser visto na tabela a seguir.

Tabela 2 - Parâmetros empregados no teste dos servidores HTTP

\begin{tabular}{l|c|c}
\hline Experimento & Número de requisições HTTP & Número de threads \\
\hline 1 & 50 & 4 \\
2 & 100 & 4 \\
3 & 50 & 8 \\
4 & 100 & 8 \\
\hline
\end{tabular}

Fonte: Dos autores.

O número de threads ou requisições concorrentes foi definido com base no número de núcleos de CPU dos chips estudados, permitindo checar se haveria alteração de desempenho e consumo de energia com o dobro de threads.

A metodologia utilizada nos ensaios com os softwares Apache e NGINX foi exatamente a mesma. Para simular os acessos HTTP nos programas Apache e NGINX foi utilizado o software Apache Bench (APACHE, 2017) que foi executado em um computador remoto conectado ao à máquina de testes via conexão local gigabit.

O Apache Bench (APACHE, 2017) têm recursos adequados aos testes pois permite configurar o número de requisições a serem solicitadas ao servidor e o nível de concorrência das requisições. 


\subsection{Metodologia para mensurar o consumo energético}

Fontes de alimentação possuem fator de eficiência variando de $60 \%$ até $80 \%$ (CORSAIR, 2012), pois parte da energia é perdida no aquecimento dos componentes internos da fonte de alimentação.

Visando mensurar apenas o consumo do próprio equipamento testado foi adotado o método de adicionar uma carga resistiva conectada a fonte de alimentação durante os ensaios.

O consumo da fonte de alimentação mais carga resistiva foi aferido previamente aos ensaios sem o sistema ligado assim se obtendo valor de consumo da fonte mais a carga dos resistores.

Durante os ensaios a carga resistiva ficou conectada a fonte em paralelo com o sistema testado. Após os testes o consumo fixo da fonte conjuntamente com a carga resistiva foi subtraído do consumo em watts aferido, assim obtendo-se somente o consumo do sistema testado.

A fonte utilizada no computador x86 foi uma fonte ATX da marca PC TOP modelo FAPT200V2 de $200 \mathrm{~W}$ reais (TORRES, 2013). A essa fonte foi adicionado um conjunto de resistores dissipando $120 \mathrm{~W}$ conectados as linhas de $12 \mathrm{~V}$ e $5 \mathrm{~V}$ como pode ser conferido na Figura 5.

Figura 5 - Diagrama do circuito resistivo conectado à fonte ATX do sistema x86

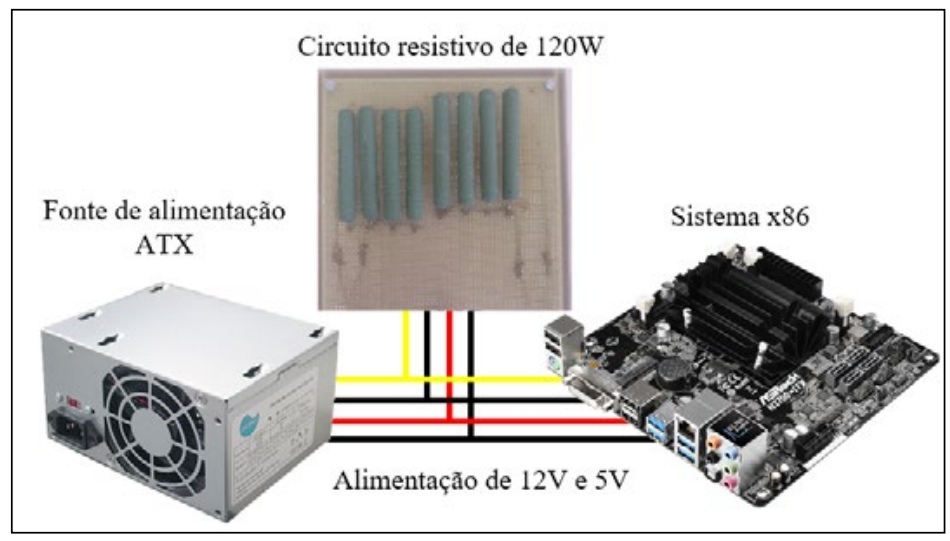

Fonte: Dos autores.

O equipamento Nvidia Shield TV possui fonte de alimentação proprietária de $40 \mathrm{~W}$ que fornece $19 \mathrm{~V}$. O circuito resistivo conectado ao Shield consome $8,3 \mathrm{~W}$. Foram empregadas cargas resistivas de consumo diferente pelo fato que a capacidade das fontes é díspar.

A fonte ATX utilizada pode fornecer até $200 \mathrm{~W}$ enquanto a fonte do equipamento Nvidia Shield TV fornece até 40W. 


\subsection{Hardware para medição elétrica}

A medição do consumo de energia dos sistemas sob análise exigia a coleta periódica de dados de tensão e corrente na alimentação do sistema de entrada da rede elétrica. Com esta finalidade foi desenvolvido um hardware próprio de medição e coleta de dados.

$\mathrm{O}$ consumo instantâneo de energia durante os ensaios foi registrado pelo transformador de corrente ZMCT103C. O transformador de corrente é um indutor que reduz a corrente elétrica passante pelo condutor, para um nível menor que possa ser mensurada por um equipamento eletrônico.

A partir deste transformador foi possível realizar a medida de corrente por uma placa Arduino Mega. A placa Arduino foi programada para medir, calcular e enviar periodicamente o consumo em watts para um computador.

A topologia de conexão do hardware desenvolvido pode ser visualizada na Figura 6.

Figura 6 - Sistema utilizado para mensurar o consumo de energia

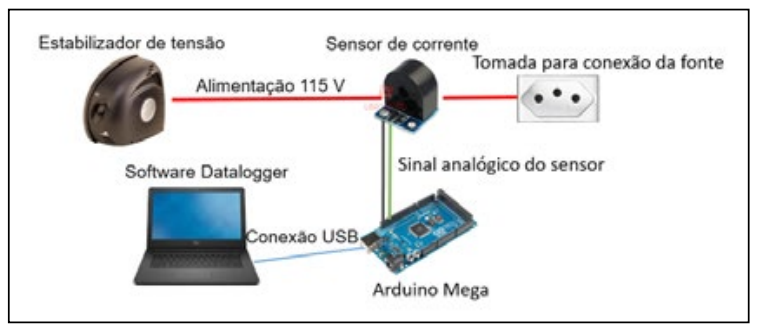

Fonte: Dos autores.

O consumo instantâneo foi registrado a cada segundo, permitindo obter o total de energia gasto por cada tarefa executada.

\section{RESULTADOS}

Nesta sessão são apresentados os resultados mensurados durante os ensaios. A apresentação dos dados aferidos está dividida pelo programa HTTP, no caso o Apache e o NGINX.

\subsection{Ensaios com servidor Apache}

Os resultados obtidos com a análise do servidor Apache são mostrados na Figura 7.

É possível constatar nos ensaios que o SOC Nvidia Tegra X1 apresentou desempenho superior ao SOC Pentium N3700 baseado na arquitetura Intel x86. Os ensaios de desempenho com o software Apache não indicaram diferença significativa de desempenho entre os ensaios com 4 e 8 threads $\mathrm{O}$ desempenho 
apresentado pelos testes com 100 requisições demostraram que o processador Tegra X1 foi $14 \%$ mais rápido que o processador Intel.

O mesmo percentual de vantagem para Arquitetura ARM foi verificado no teste com 50 requisições. Durante os testes de desempenho o uso de CPU de ambos os sistemas foi próximo de 100\% em todos os testes em ambos os sistemas analisados.

Figura 7 - Desempenho do software Apache

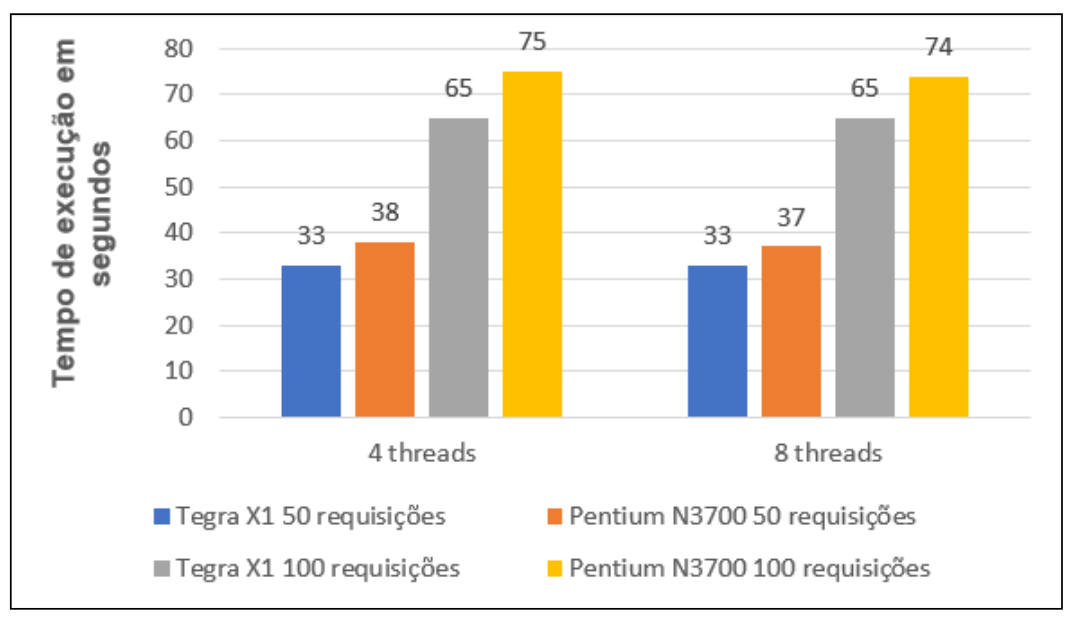

Fonte: Dos autores.

Os ensaios de consumo utilizando o software Apache demostraram que o processador Pentium N3700 foi de $8 \%$ a $11 \%$ mais eficiente que o processador Tegra X1 em todos os 4 experimentos efetuados conforme pode ser conferido na Figura 8. 
Figura 8 - Consumo de energia durante os testes com o Apache

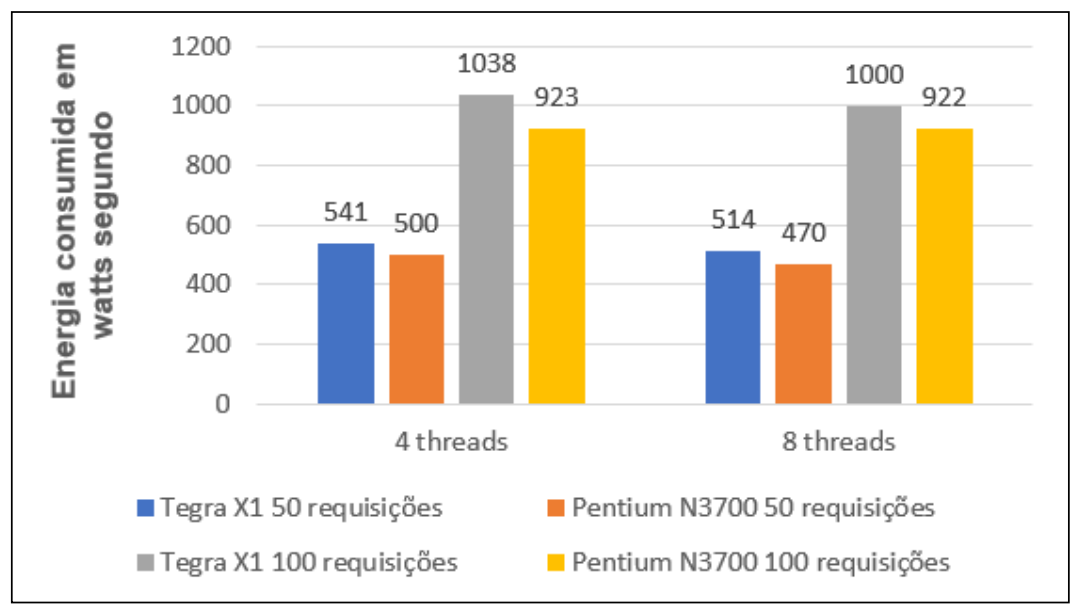

Fonte: Dos autores.

Os dados da Figura 8 demonstram que não houve diferença significativa dos testes de 100 e 50 requisições executados nas variantes de 4 e 8 requisições simultâneas.

A média do consumo do sistema baseado em ARM executando os testes com o software apache foi de 15,9W enquanto que na plataforma X86 foi de $12,3 \mathrm{~W}$.

\subsection{Ensaios com servidor NGINX}

A metodologia empregada para mensurar o desempenho e eficiência energética no software NGINX foi a mesma utilizada no programa Apache.

Os resultados indicam que o SOC Tegra X1 apresentou desempenho 8\% superior quando comparado ao Pentium N3700, nos ensaios de 50 requisições e nos ensaios com 100 requisições o processador Tegra X1 apresentou desempenho $12 \%$ superior ao processador $x 86$ conforme pode ser conferido na Figura 9. 
Figura 9 - Desempenho do software NGINX

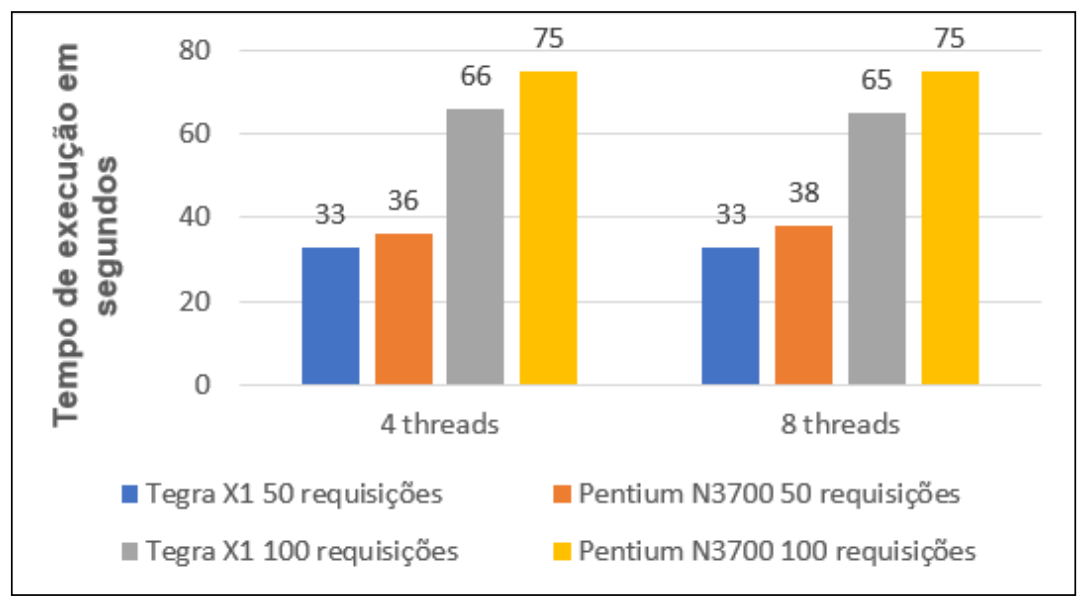

Fonte: Dos autores.

Não houve diferença significativa nas variantes dos testes com 50 e 100 requisições simultâneas, assim como obtido nos dados no teste do Apache. Durante os ensaios foi verificado que o uso de CPU ficou próximo de $100 \%$ nos dois computadores utilizados.

Ao analisar o consumo de energia mensurado na Figura 10 foi constatado que o SOC Pentium N3700 foi aproximadamente 16\% mais econômico nos ensaios com 100 requisições e aproximadamente $20 \%$ mais econômico nos ensaios com 50 requisições conforme figura a seguir.

Figura 10 - Consumo de energia durante os testes com o NGINX.

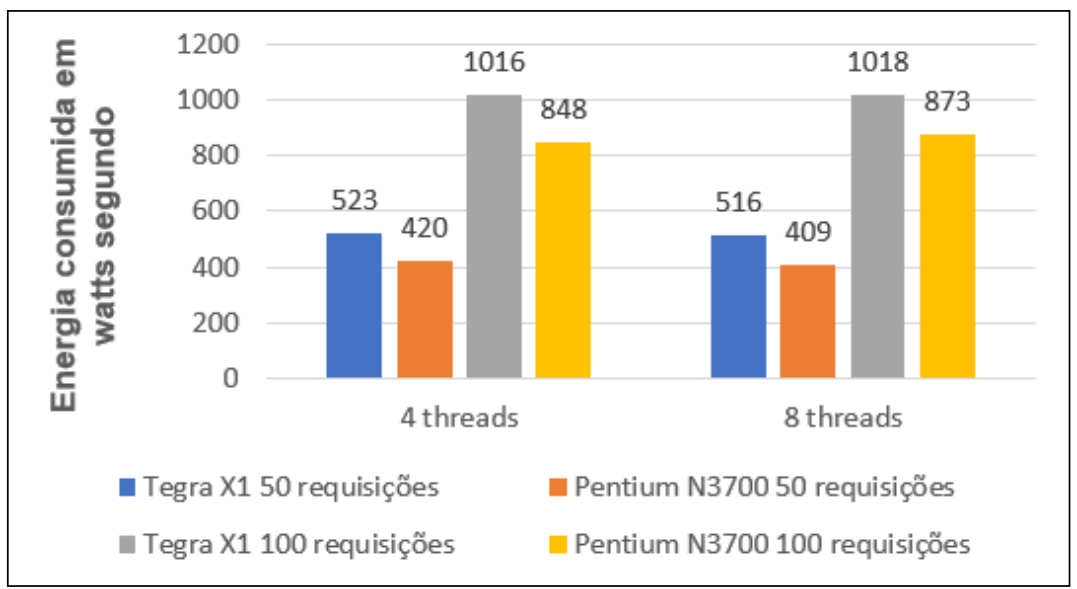

Fonte: Dos autores. 
Segundo os dados da Figura 10 não se percebe diferença significativa de consumo nos ensaios de 4 e 8 threads, pois houve pouca variação no consumo total de teste para teste.

\section{DISCUSSÃO DOS RESULTADOS OBTIDOS}

A metodologia e os materiais utilizados nos ensaios permitiram mensurar o desempenho e consumo energético da microarquitetura ARM Cortex A57 perante a arquitetura Intel x86.

O tempo de execução de um mesmo teste difere nas duas plataformas estudadas, considerando essa circunstância o tempo não foi levado em consideração no cálculo de eficiência energética.

Visando calcular a eficiência energética foi utilizado o dado (W.s) obtido através da divisão no consumo acumulado em watts do teste pelo número de requisições do teste efetuado.

Para efetuar a comparação (W.s) por requisição foi utilizado somente o teste de 100 requisições por 8 threads simultâneas; os resultados podem ser conferidos na Tabela 3.

Tabela 3 - Consumo em Watts por cada requisição HTTP ou query executada

\begin{tabular}{l|l|l}
\hline Software & Tegra X1 & Pentium N3700 \\
\hline Apache & $10 \mathrm{Ws}$ & $9,92 \mathrm{Ws}$ \\
\hline NGINX & $10,8 \mathrm{Ws}$ & $8,73 \mathrm{Ws}$ \\
\hline
\end{tabular}

Fonte: Dos autores.

Observa-se na Tabela 3 que a diferença de eficiência verificada no SOC Tegra X1 em relação ao chip Pentium N3700 foi menor que 1\% a favor do processador da Intel, resultado que pode ser atribuído a energia total consumida pelos dois sistemas para executar o teste de 100 requisições com 8 threads

O processador Pentium N3700 por sua vez foi $19 \%$ mais eficiente no consumo de energia que o chip da Nvidia utilizado. A diferença do resultado é devido ao fato que consumo de energia do Pentium foi menor para completar o processamento das 100 requisições mesmo demorando mais tempo para completar a tarefa.

O desempenho superior obtido no sistema ARM pode ser proveniente da velocidade superior da memória LPDDR 4 do Nvidia Shield TV (2400 MHz), em relação a memória DDR3 $1600 \mathrm{MHz}$ do sistema x86. As plataformas testadas foram configuradas de forma que o gargalo de desempenho fosse o CPU e a memória; sendo que a velocidade da memória não pode ser desprezada nesse caso. 
Assim como os resultados os verificados pelos autores Aroca e Gonçalves (2012), a arquitetura ARM continua apresentando desempenho superior a arquitetura x86 em CPUs de baixo consumo energético. Em relação ao o resultado encontrado neste trabalho foi diferente do artigo publicado em 2012 apontando para a arquitetura x86 energeticamente mais eficiente; tal resultado pode ser atribuído ao fato que o processador Atom N280 utilizado na pesquisa anterior não era um SOC, necessitando de um chip do tipo nortbridge para gerenciar acesso a memória RAM, acesso a disco e conexão de rede aumentando o consumo de energia; esse resultado indica que sistemas baseados em SOCs são mais eficientes.

\section{CONCLUSÕES}

A pesquisa efetuada por esse trabalho possibilitou mensurar e avaliar de forma prática o desempenho e eficiência energética do SOC Nvidia Tegra X1 baseado na microarquitetura ARM Cortex A57 em relação ao chip Intel Pentium N3700.

Após avaliar os dados é possível afirmar que o chip Tegra X1 é aproximadamente $12 \%$ mais rápido na execução de páginas WEB escritas na linguagem PHP sendo executados nos servidores HTTP Apache e NGINX.

Os dados obtidos de eficiência energética nos ensaios com os programas Apache e NGINX indicam que o SOC Pentium N3700 é mais eficiente em cerca de até $19 \%$.

Os resultados mensurados demostraram que os processadores ARM mais recentes podem competir com processadores Intel x 86 de baixo consumo em desempenho, permitindo o seu uso em computadores mais complexos como servidores. Levando em consideração o trabalho publicado por Aroca e Gonçalves (2012), pode ser verificado que a Intel tem avançado bastante em desenvolver CPUs energeticamente mais eficientes, considerando que o sistema baseado no Pentium N3700 apresentou eficiência energética superior à o Nvidia $\mathrm{X} 1$, tal característica é relevante para utilização em servidores, notebooks e computadores embarcados.

\section{REFERÊNCIAS}

ANTHONY Sebastian. SoC vs. CPU - The battle for the future of computing.2012. Disponível em:<http:/ /www.extremetech.com/computing/126235-soc-vs-cpu-thebattle-for-the-future-of-computing > Acesso em: 25 de abr. 2016.

APACHE. ab - Apache HTTP server benchmarking tool. 2017. Disponível em: < https://httpd.apache.org/docs/2.4/programs/ab.html> Acesso em: 12 dez. 2017.

ARM.Cortex-A57 Processor.2016. Disponível em: <http:/ /www.arm.com/products/ processors/cortex-a/cortex-a57-processor.php> Acesso em: 24 abr. 2016. 
AROCA, Rafael Vidal; GONÇALVES, Luiz Marcos G. Towards green data centers: A comparison of $x 86$ and ARM architectures power efficiency. 2012. Disponível em: $<$ https://www.researchgate.net/publication/232713208_Towards_green_data_ centers_A_comparison_of_x86_and_ARM_architectures_power_efficiency > Acesso em 06 jun. 2016

ASROCK. N3700-ITX. 2015. Disponível: < http:/ / www.asrock.com/mb/Intel/N3700ITX/> Acesso em: 08 dez. 2017.

BLEM, Emily et al. A Detailed Analysis of Contemporary ARM and x86 Architectures. (2013). Disponível em: <https:/ /www.researchgate.net/ publication/266457125_A_Detailed_Analysis_of_Contemporary_ARM_and_x86_ Architectures> Acesso em 08 dez. 2017

CORSAIR. 80 PLUS Platinum efficiency; What does it mean, and what's the benefit to me?. 2012. Disponível em: http:/ / www.corsair.com/en-us/blog/2012/august/80plus-platinum-what-does-it-mean-and-what-is-the-benefit-to-me> Acesso em: 08 nov.2016.

FRUMUSANU Andrei, SMITH Ryan. ARM A53/A57/T760 investigated - Samsung Galaxy Note 4 Exynos Review. 2015. Disponível: em < http:/ / www.anandtech.com/ show/8718/the-samsung-galaxy-note-4-exynos-review/ > Acesso em: 30 maio. 2016.

INTEL. Intel ${ }^{\circledR}$ Pentium ${ }^{\circledR}$ Processor N3700. $2015<$ http:/ / ark.intel.com/Pt-Br/ products/87261/Intel-Pentium-Processor-N3700-2M-Cache-up-to-2_40-GHz> Acesso em: 08 nov. 2016.

Intel® Xeon ${ }^{\circledR}$ Processor E7-4820 v4. $2016<$ https: / / ark.intel.com/ products/93814/Intel-Xeon-Processor-E7-4820-v4-25M-Cache-2_00-GHz> Acesso em: 15 dez. 2017.

. Products (Formerly Avoton). 2013. Disponível em:<http:/ /ark.intel.com/ products/codename/54859/Avoton> Acesso em: 9 abr. 2016.

KINGSTON. SDHC/SDXC UHS-I Classe 10 - 16GB-512GB I Kingston. 2016. Disponível em: <https://www.kingston.com/br/flash/sd_cards/sda10> Acesso em: 08 mai. 2016.

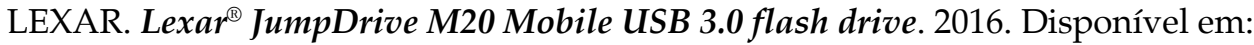
<http:/ / www.lexar.com/products/usb-flash-drives/JumpDrive-M20-Mobile-USB-3Flash-Drive.html\#SKU=LJDM20-64GBBNL> Acesso em: 08 nov. 2016.

NETCRAFT. April 2016 Web Server Survey.2016 Disponível em:<http:/ / news. netcraft.com/archives/2016/04/21/april-2016-web-server-survey.html> Acesso em: 12 abr.2016.

NVIDIA. NVIDIA SHIELD. 2016. Disponível em: <https:/ / shield.nvidia.com/ android-tv> Acesso em 29 mai. 2016. 
NVIDIA. Processadores Tegra. 2015. Disponível em: <http:/ / www.nvidia.com.br / object/tegra-x1-processor-br.html>Acesso em: 4 mar. 2016.

SMITH, Ryan. NVIDIA Announces SHIELD Console: Tegra X1 Android TV Box Shipping in May. 2015. Disponível em: <http:/ / www.anandtech.com/show/9047/ nvidia-announces-shield-console-tegra-x1-android-tv-box-shipping-in-may $>$ Acesso em: 08 nov. 2016.

TORRISI, Alessandro. Free PHP Benchmark Performance Script. 2012. Disponível em: <http:/ / www.php-benchmark-script.com/>Acesso em: 5 dez. 2017.

TORRES, Gabriel. Teste da fonte de alimentação PCTop FAPT200. 2013. Disponível em: <http:/ / www.clubedohardware.com.br/artigos/teste-da-fonte-de-alimentacaopctop-fapt200/2787> Acesso em: 08 nov. 2016.

TOYOTARO, Suzumura. et al. Performance Comparison of Web Service Engines in PHP, Java, and C. In 2008 IEEE International Conference on Web Services, 2008 Performance Script. Disponível em: <http:/ / ieeexplore.ieee.org/ document/4670199/?reload=true> (2010); acesso em: 08 maio. 2017. 